\title{
A Arte no Ensino da Cardiologia: Relato da Experiência do Uso de Massas Moldáveis no Aprendizado da Anatomia Normal e Patológica do Coração
}

\section{Learning Cardiology through Art: Modeling Hearts to Understand Normal and Abnormal Anatomy}

Raimundo Francisco de Amorim Junior ${ }^{I}$

Rossana Severi ${ }^{I}$

Lucia Didier Moser

Sandra da Silva Mattos ${ }^{I}$

\section{PALAVRAS-CHAVE}

- Cardiologia

- Cardiopatias Congênitas.

- Medicina na Arte.
INTRODUÇÃO: A anatomia e a fisiologia do coração humano há muito tempo despertam curiosidade, dada a complexidade da morfologia cardíaca, a sua importância para a manutenção da vida e seu grande valor simbólico. O entendimento da fisiologia cardíaca e sua representação nas diversas modalidades artísticas têm ajudado até hoje na formação de profissionais de saúde que trabalham com cardiologia e também na comunicação com familiares. Este artigo apresenta a experiência de um especializando em um serviço de referência no ensino de cardiologia pediátrica, na construção de modelos de corações normais e patológicos em massa de modelar e massa de biscuit, e as implicações no processo de ensino-aprendizagem do grupo de preceptores e residentes da instituição. RELATO DE EXPERIÊNCIA: Um residente do primeiro ano de cardiologia pediátrica, sem treinamento ou experiência prévia com artes plásticas, foi estimulado pela preceptoria do programa a moldar corações normais e com cardiopatias congênitas para fins de aprendizado da anatomia interna e externa do órgão. Os materiais utilizados foram massa de modelar e massa de biscuit. RESULTADOS: Dez peças foram produzidas ao longo de um ano de especialização, com melhora progressiva nos aspectos artísticos e anatômicos. Entre os modelos patológicos, foram produzidas peças representativas de tetralogia de Fallot, transposição dos grandes vasos, persistência do canal arterial e comunicação interventricular. CONCLUSÃO: A experiência proporcionou três efeitos positivos constatados pela equipe: maior entendimento da anatomia cardíaca normal e patológica por parte do especializando envolvido, o qual se refletiu, por exemplo, em melhor compreensão de imagens médicas, como ecocardiografia, tomografia computadorizada e ressonância magnética cardíacas, maior aproximação dos residentes do programa com as artes plásticas em uma especialidade que sempre teve uma relação muito estreita com o desenho e a escultura, e produção de modelos em três dimensões de estruturas normais e patológicas que podem ser usadas em aulas e encontros científicos para discussão da disciplina. 


\section{KEY-WORDS}

- Cardiology.

- Heart Defects, Congenital

- Medicine in the Arts.

Recebido em: 10/4/18

Aceito em: 18/7/18
ABSTRACT

INTRODUCTION: The anatomy and physiology of the human heart has long captivated the curiosity of human beings, due to its complexity, its importance for maintaining life, and its great symbolic value. The understanding of cardiac physiology and its representations in different art forms has assisted in the training of health professionals, particularly those working in cardiology, as well as communication with families. This article presents the experience of a pediatric cardiology resident using modeling clays to build normal and abnormal heart models, and discusses the implications of this practice in the teaching-learning process among staff and trainees of the institution. CASE REPORT: A first year paediatric cardiology resident, with no previous training or experience in the plastic arts, was encouraged by Head of Department to mould hearts with normal anatomy and with congenital heart disease for the purpose of teaching the internal and external anatomy of the organ. The materials used were plasticine and cold porcelain clay. RESULTS: Ten anatomical pieces were produced over a one-year period, with progressive improvements in artistic and anatomical aspects. Among the pathological models, there were pieces demonstrating the tetralogy of Fallot, transposition of the great vessels, patent ductus arteriosus, and ventricular septal defect. CONCLUSION: The experience lead to three positive effects noted by the medical team: a better understanding of normal and abnormal cardiac anatomy by the residents, evidenced by their rapid improvement in interpreting medical images such as echocardiography, computerized tomography and nuclear magnetic resonance images; greater access of residents to the plastic arts, in a medical specialty that has always benefited from close proximity with drawings and sculpture; and the production of very low-cost 3D models of normal and abnormal cardiac anatomy that can be used for lectures, clinical-surgical and scientific meetings, and teaching future students.

\section{INTRODUÇÃO}

A anatomia e a fisiologia do coração humano há muito tempo despertam curiosidade, dada a complexidade da morfologia desse órgão, a sua importância para a manutenção da vida e seu grande valor simbólico, derivados de características como a pulsatilidade e sua localização central no corpo humano. $\mathrm{Na}$ tentativa de compreender o coração ou reproduzir simbolicamente o órgão, artistas têm se utilizado do desenho, da pintura e da escultura desde a pré-história até os dias atuais ${ }^{1-3}$.

O entendimento da anatomia cardíaca e sua representação nas diversas modalidades artísticas têm ajudado até hoje na formação de profissionais de saúde que trabalham com cardiologia e também na comunicação com familiares. Na ausência de peças anatômicas adequadas, o estudo da anatomia cardíaca é extremamente dependente de representações artísticas em duas e três dimensões, como desenhos e gráficos, além de imagens ecocardiográficas, tomográficas, etc. ${ }^{4}$. Além desses usos, é estudado o impacto de intervenções artísticas no tratamento de pacientes com cardiopatia, sobretudo crianças e adolescentes ${ }^{5-7}$.

O surgimento de métodos modernos de imagem, simuladores e modelos nos permite questionar se ainda há espaço para a arte no ensino médico. Poucos trabalhos e estudos de caso demonstram que estudantes de Medicina tiram proveito de intervenções que aproximem o ensino da anatomia e as manifestações artísticas, sobretudo o desenho e a pintura ${ }^{8-11}$.

Existem ainda poucos trabalhos na literatura acerca da influência da escultura de materiais como argila, massa de modelar e cera no processo de ensino-aprendizagem na área de saúde, nenhum deles com foco específico no ensino da cardiologia $^{12-14}$.

Este trabalho relata a experiência de um serviço de referência no ensino de cardiologia pediátrica que estimulou um especializando sem treinamento prévio em artes plásticas a construir modelos de corações normais e patológicos em massa de modelar e massa de biscuit, e as implicações no processo de ensino-aprendizagem do grupo de preceptores e residentes da instituição.

\section{RELATO DE EXPERIÊNCIA}

Um residente do primeiro ano de cardiologia pediátrica sem treinamento ou experiência prévia com escultura foi estimulado pela preceptoria do programa a moldar corações normais e 
com cardiopatias congênitas para fins de aprendizado da anatomia interna e externa do órgão. O material utilizado inicialmente foi massa de modelar.

Os dois primeiros modelos fabricados foram um corte apical quatro câmaras e um corte que demonstra a via de saída do ventrículo esquerdo (Figura 1). Nota-se a preocupação com as relações entre as cavidades e as estruturas cardíacas entre si, como o posicionamento da valva atrioventricular direita em situação mais apical, a banda moderadora no ventrículo direito e a continuidade fibrosa mitro-aórtica.
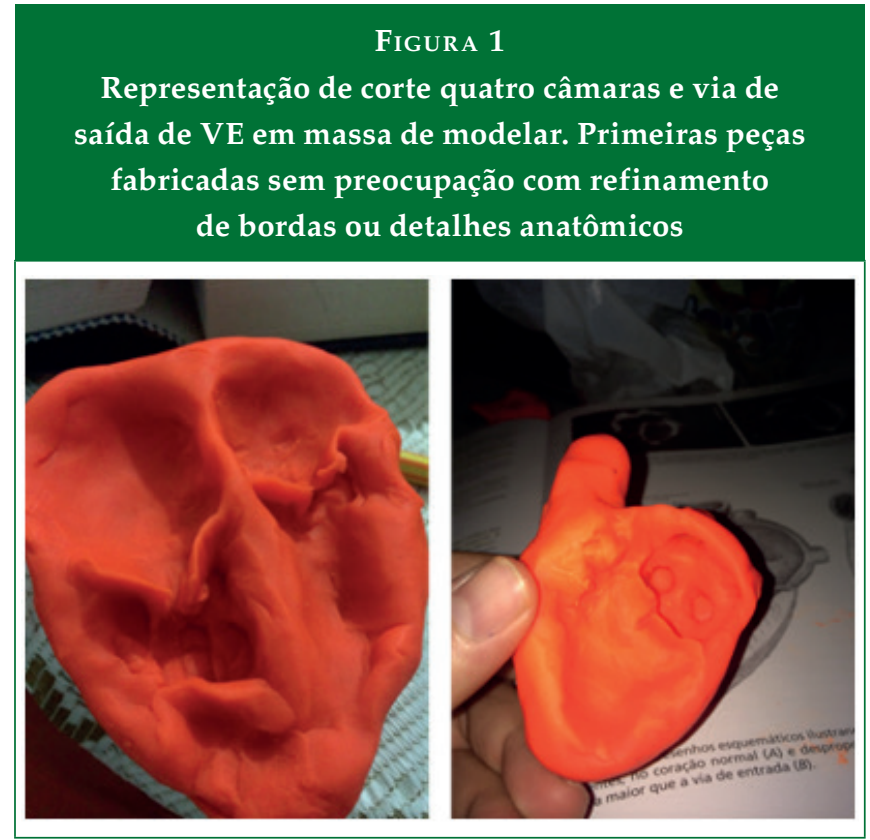

Após os primeiros trabalhos, manteve-se o estímulo positivo para a manutenção da produção artística. Com a experiência, os modelos subsequentes apresentaram melhor qualidade técnica e mais precisão nos detalhes anatômicos (Figura 2). Algumas peças, uma vez finalizado o aspecto externo do coração, eram cortadas para reproduzir a anatomia interna do órgão (Figura 3).

Os modelos em massa de modelar não apresentavam boa durabilidade, uma vez que se deformavam facilmente durante a manipulação e o transporte. A maioria das peças construídas com este material foi desprezada. O passo seguinte, portanto, foi procurar um material que permitisse maior durabilidade dos modelos, tendo sido escolhida a massa de biscuit, que, após seca, adquire consistência rígida. A mudança permitiu o transporte e a exposição das peças em demonstrações e aulas.

Peças de órgãos com defeitos cardíacos congênitos, como comunicação interventricular, canal arterial patente, transpo-
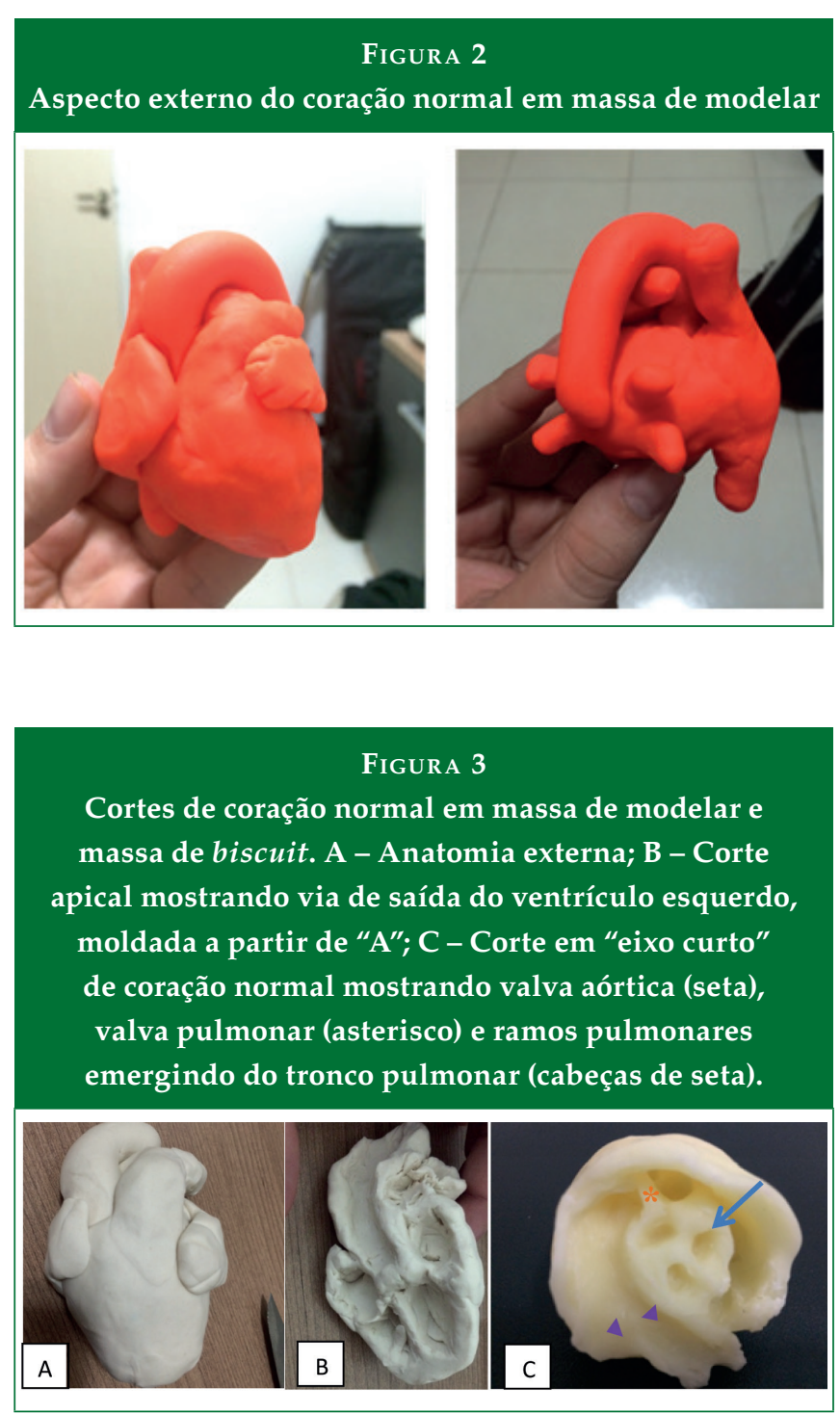

sição dos grandes vasos da base e tetralogia de Fallot, também foram moldadas em massa de biscuit (Figura 4).

O trabalho proporcionou resultados além do esperado, promovendo ao menos três efeitos positivos:

- Maior entendimento da anatomia cardíaca normal e patológica por parte do estudante e dos seus pares, que analisaram e opinaram na criação das peças, além de maior familiaridade com as estruturas e defeitos cardíacos e sua posição espacial no órgão, o que se refletiu, por exemplo, em melhor compreensão de imagens médicas, como ecocardiografia, tomografia computadorizada e ressonância magnética cardíacas pelos residentes;

- Maior aproximação entre os residentes do programa e as artes plásticas numa especialidade que sempre teve relação muito estreita com o desenho e a escultura; 

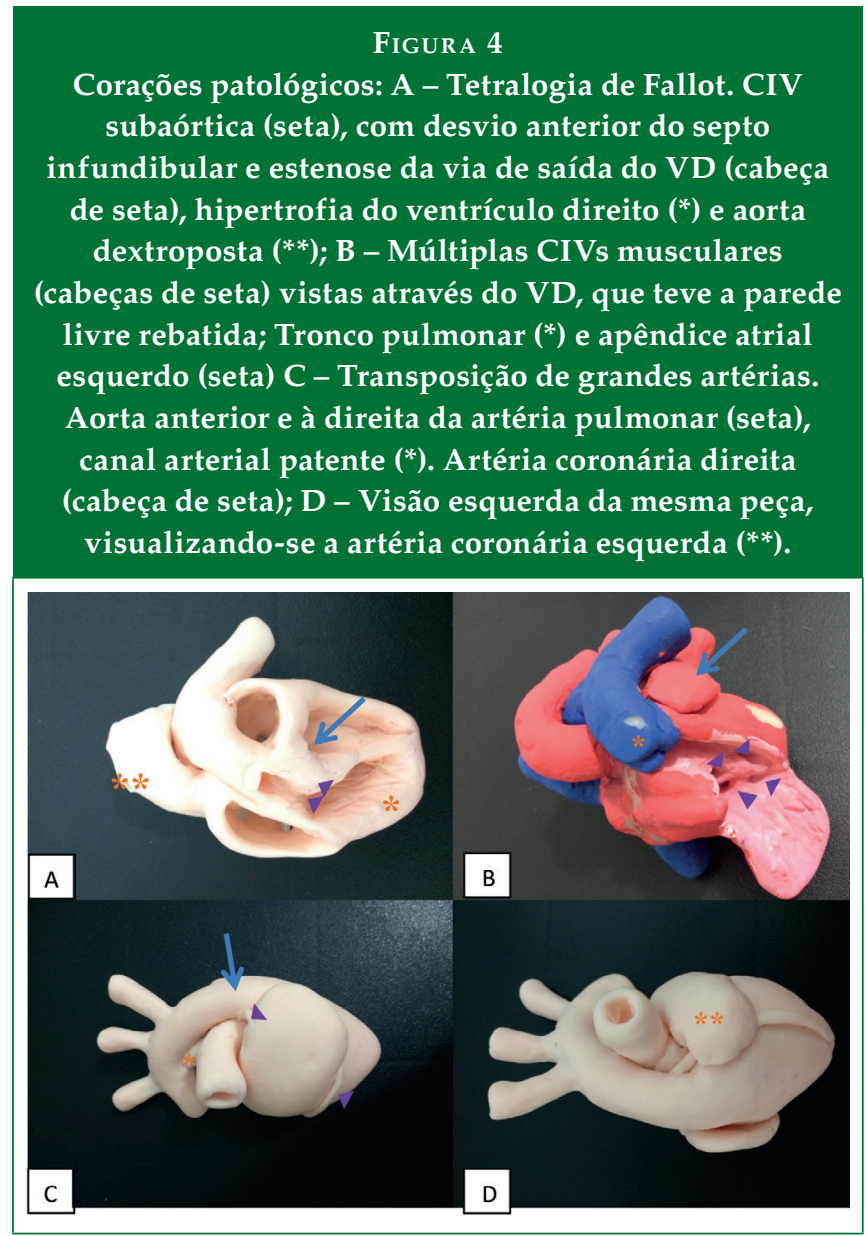

- Produção de modelos em três dimensões de estruturas normais e patológicas que poderão ser usadas em aulas e encontros científicos para discussão da disciplina.

\section{DISCUSSÃO}

A representação mais antiga de um coração de mamífero data de 30 mil anos atrás, do período Paleolítico, numa imagem pintada em vermelho no tórax de um elefante, na caverna Pindal, em Astúrias, na Espanhaํ. Os egípcios, que usavam amplamente o coração em sua mitologia, tendo sido um dos primeiros povos a considerar o coração como "o centro da alma", também legaram ao mundo gravuras e hieroglifos, datados de até 2000 a.C., com corações humanos ${ }^{1,15}$. A primeira representação de um coração com preocupação anatômica data de 1200-900 a.C., sendo uma escultura do povo Olmec, que habitou o México, mostrando um coração humano dividido em duas câmaras, com três grandes vasos emergindo da peça que se apoia numa figura humana desproporcionalmente pequena ${ }^{1,16}$.
Outro grande marco no uso da arte para entendimento do coração humano se dá na Renascença europeia, sobretudo nas obras de Leonardo da Vinci e, pouco tempo depois, com a obra-prima de Andreas Versalius, De Humanis Corporis Fabrica. Da Vinci foi um dos primeiros anatomistas a retratar o coração com quatro câmaras cardíacas e detalhou a anatomia das valvas semilunares e da circulação coronariana. É provável que Versalius tenha travado conhecimento com seu trabalho, uma vez que escreveu o clássico De Humanis Corporis Fabrica 21 anos após o falecimento de Da Vinci. Não há registros, entretanto, de que tenha havido tal contato ${ }^{3,17,18}$.

No campo das cardiopatias congênitas, a arte sempre foi a maior aliada no entendimento dos aspectos, por vezes bastante complexos, que norteiam as inúmeras variações da anatomia normal. No século XIX, grandes anatomistas, como John Richard Farré, Karl, Freiherr von Rokitansky, John Keaing e William Edwards, descreveram a circulação fetal e ilustraram anomalias como truncus arteriosus, tetralogia de Fallot e coarctação de aorta com tal riqueza de detalhes que impressionam até os dias de hoje ${ }^{19}$.

A habilidade e o treinamento nas mais diversas modalidades artísticas são características compartilhadas pela maioria dos grandes anatomistas do passado e também da era moderna, como Frank Netter. Seus trabalhos têm auxiliado até hoje o ensino da anatomia humana normal e patológica.

A escultura também teve papel importante na demonstração de corações normais e patológicos, com destaque para as esculturas em cera. Algumas peças são armazenadas e expostas em universidades, e alguns autores questionam se elas possuem hoje maior valor artístico ou científico, dada a beleza e o realismo das peças ${ }^{20}$. Existem relatos de uso da massa de modelar no ensino da anatomia. $\mathrm{O}$ material pode ser usado para estudar a anatomia descritiva, topográfica e segmentação de diversos órgãos. Quando comparadas com outros métodos que não a análise da peça anatômica de cadáver, as intervenções descritas na literatura têm se mostrado benéficas ao aprendizado ${ }^{12,14}$.

Atualmente, diversos métodos de análise da anatomia cardíaca são disponíveis, além das ilustrações médicas e do estudo de peças anatômicas, como imagens cardíacas em alta definição de ecocardiograma bi- e tridimensional, tomografia e ressonância magnética cardíacas, simuladores e ferramentas audiovisuais. Entre estes, destacam-se os métodos que permitem visualizar o coração em três dimensões, como a reconstrução em 3D de imagens cardíacas. É descrita a dificuldade de estudantes e profissionais em treinamento em cardiologia em elaborar uma imagem mental em três dimensões do órgão, uma vez que o cérebro humano tem dificuldades em reconhe- 
cer em três dimensões objetos com formatos aos quais não está habituado ${ }^{21-23}$.

Na cardiologia infantil, bem como em outras áreas, a impressão em três dimensões de modelos normais e patológicos, a partir de exames de tomografia, ressonância magnética e, mais recentemente, ecocardiografia $3 \mathrm{D}$, vem sendo usada para o ensino da anatomia e correlações clínico-cirúrgicas com bons resultados ${ }^{4,22,24}$

A experiência de construir modelos em massa de modelar e biscuit para simular corações normais e com cardiopatias congênitas mostrou-se extremamente proveitosa para o aprendizado da disciplina e ajudou sobremaneira os envolvidos a compreender as relações anatômicas das câmaras cardíacas entre si e com os grandes vasos da base além da circulação coronariana.

Foi percebido também que, a partir de um modelo normal bem construído e trabalhado, é possível predizer e moldar diversas anomalias congênitas, como comunicação interatrial e interventricular, tetralogia de Fallot, transposição dos grandes vasos da base, persistência do canal arterial e coarctação da aorta. Por vezes, moldar o defeito torna-se intuitivo quando se está construindo o modelo.

É possível também concluir que, mesmo sem treinamento formal em artes plásticas, não existe impedimento para a construção de modelos de relativa semelhança com a peça anatômica, sendo os materiais usados de fácil acesso e de baixo custo.

Apesar dos avanços tecnológicos na área e do grande potencial que guardam para o ensino da cardiologia, a arte parece andar junto da disciplina e ainda continuará auxiliando a formação de novos profissionais, bem como continuará a produzir grandes obras para o deleite dos olhos e do imaginário de todos aqueles que trabalham com cardiologia.

\section{REFERÊNCIAS}

1. Bendersky G. The Olmec Heart Effigy: Earliest Image od the Human Heart. Perspect Biol Med. 1997;40(3):348-61.

2. Bestetti RB, Restini CBA, Couto LB. Development of anatomophysiologic knowledge regarding the cardiovascular system: from Egyptians to Harvey. Arq Bras Cardiol [Internet]. 2014;103(6):538-45. Available from: http://www. pubmedcentral.nih.gov / articlerender.fcgi?artid $=4290745$ \&tool=pmcentrez\&rendertype $=$ abstract

3. Mesquita ET, Souza Júnior CV de, Ferreira TR. Andreas Vesalius 500 years - A Renaissance that revolutionized cardiovascular knowledge. Rev Bras Cir Cardiovasc [Internet]. 2015;260-5. Available from: http://www.gnresearch. org/doi/10.5935/1678-9741.20150024
4. Costello JP, Olivieri LJ, Krieger A, Thabit O, Blair Marshall M, Yoo S-J, et al. Utilizing Three-Dimensional Printing Technology to Assess the Feasibility of High-Fidelity Synthetic Ventricular Septal Defect Models for Simulation in Medical Education.

5. Wang Q, Hay M, Clarke D, Menahem S. Adolescents' drawings of their cardiac abnormality. Cardiol Young [Internet]. 2011;21(5):556-61. Available from: http:/ / www.ncbi. nlm.nih.gov/pubmed/21554829

6. Hatem TP, Lira PIC, Mattos SS. The therapeutic effects of music in children following cardiac surgery. J Pediatr (Rio J). 2006;82(3):186-92.

7. Dolidze K, Smith EL, Tchanturia K. A clinical tool for evaluating emotional well-being: Self-drawings of hospitalized children. J Pediatr Nurs [Internet]. 2013;28(5):470-8. Available from: http:/ / dx.doi.org/10.1016/j.pedn.2013.02.026

8. Bell LTO, Evans DJR. Art, anatomy, and medicine: Is there a place for art in medical education? Anat Sci Educ [Internet]. 2014 Sep 10;7(5):370-8. Available from: http:/ / doi. wiley.com/10.1002/ase.1435

9. Phillips PS. Running a life drawing class for pre-clinical medical students. Med Educ. 2000;34(12):1020-5.

10. Pickering JD. Taking human anatomy drawings for teaching outside the classroom. Surg Radiol Anat. 2014;36(9):953-4.

11. Liou KT, George P, Baruch JM, Luks FI. Clinical Sketches: teaching medical illustration to medical students. Med Educ. 2014;48(5):525.

12. Myers DL, Arya LA, Verma A, Polseno DL, Buchanan EM. Pelvic anatomy for obstetrics and gynecology residents: An experimental study using clay models. Obstet Gynecol. 2001;97(2):321-4.

13. Waters JR, Van Meter P, Perrotti W, Drogo S, Cyr RJ. Cat dissection vs. sculpting human structures in clay: an analysis of two approaches to undergraduate human anatomy laboratory education. Adv Physiol Educ. 2005;29(1):27-34.

14. Oh CS, Kim JY, Choe YH. Learning of cross-sectional anatomy using clay models. Anat Sci Educ. 2009;2(4):156-9.

15. Ferrari R. The Story of the Heartbeat II. Eur Heart J. 2012;33(2):152-3.

16. Phoon CK. The Olmec heart effigy revisited: A highly accurate, ancient depiction of the human heart. Perspect Biol Med [Internet]. 1998;41(4):540-8. Available from: http:/ / search.proquest.com/docview / 233166166?accountid=15533

17. Shoja MM, Agutter PS, Loukas M, Benninger B, Shokouhi G, Namdar H, et al. Leonardo da Vinci's studies of the heart. Int J Cardiol [Internet]. 2013;167(4):1126-33. Available from: http:/ /dx.doi.org/10.1016/j.ijcard.2012.09.078 
18. Sterpetti A V. The revolutionary studies by Leonardo on blood circulation were too advanced for his times to be published. J Vasc Surg [Internet]. 2015;62(1):259-63. Available from: http:/ /dx.doi.org/10.1016/j.jvs.2015.03.036

19. Evans WN, Acherman RJ, Anderson RH. One hundred years of texts describing congenitally malformed hearts from 1814 to 1914. Cardiol Young [Internet]. 2009;19(1):1229. Available from: http://www.ncbi.nlm.nih.gov/pubmed/19079849

20. Ballestriero R. Anatomical models and wax Venuses: Art masterpieces or scientific craft works? J Anat. 2010;216(2):223-34.

21. Bülthoff I, Bülthoff $H$, Sinha P. Top-down influences on stereoscopic depth-perception. Nat Neurosci. 1998;1(3):254-7.

22. Mottl-Link S, Hübler M, Kühne T, Rietdorf U, Krueger JJ, Schnackenburg B, et al. Physical Models Aiding in Complex Congenital Heart Surgery. Ann Thorac Surg. 2008;(86):273-7.

23. Wagner R, Razek V, Gräfe F, Berlage T, Janoušek J, Daehnert I, et al. Effectiveness of simulator-based echocardiography training of noncardiologists in congenital heart diseases. Echocardiography. 2013;30(6):693-8.

24. Olivieri LJ, Krieger A, Loke YH, Nath DS, Kim PCW, Sable CA. Three-dimensional printing of intracardiac defects from three-dimensional echocardiographic images: Feasibility and relative accuracy. J Am Soc Echocardiogr [Internet]. 2015;28(4):392-7. Available from: http:/ /dx.doi. org/10.1016/j.echo.2014.12.016

\section{CONTRIBUIÇÃO DOS AUTORES}

Todos contribuíram de maneira igual.

\section{CONFLITO DE INTERESSES}

Nenhum.

\section{ENDEREÇO PARA CORRESPONDÊNCIA}

Raimundo Francisco de Amorim Junior

Rua Walter Duarte Pereira, 1560

Capim Macio - Natal

CEP 59082-470 - RN

E-mail: raimundomorim@gmail.com 